Palabra Clave (La Plata), octubre 2017, vol. 7, n 1, e033. ISSN 1853-9912

Universidad Nacional de La Plata.

Facultad de Humanidades y Ciencias de la Educación.

Departamento de Bibliotecología

\title{
Perfiles de autor en repositorios institucionales
}

\section{Author profiles in institutional repositories}

\author{
Patricia Genovés * \\ * Universidad Nacional de La Plata. Facultad de Humanidades y Ciencias de la Educación. \\ Biblioteca "Profesor Guillermo Obiols", Argentina | pgenoves@fahce.unlp.edu.ar
}

\section{PALABRAS CLAVE}

Repositorios institucionales

Perfiles de autor

Perfiles de investigador

Servicios de valor agregado

Investigadores

Sistemas de información científica

Producción científica

Políticas y mandatos de acceso abierto

Argentina

\section{KEYWORDS}

Institutional repositories

Author profiles

Researcher profiles

Added-value services

Researchers

Current Research Information Systems

Scientific production

Open-access mandates and policies

\section{RESUMEN}

Este trabajo describe las funciones y utilidades de la implementación de perfiles de autor en repositorios institucionales, como un servicio de valor agregado dirigido a acrecentar su uso entre los miembros de una institución y mejorar la visibilidad de la producción científica alojada en el repositorio. Incluye un relevamiento de servicios de perfiles de autor implementados en repositorios institucionales argentinos y extranjeros. Advierte que, ante el establecimiento de mandatos nacionales de depósito, la necesidad de contar con repositorios institucionales fuertes y sostenibles en el tiempo es aún mayor que antes. Integrar a los repositorios las funcionalidades de los sistemas curriculares y de gestión de información científica y tecnológica es un camino para lograr esa fortaleza, y los perfiles de autor son el resultado visible de dicha integración. Señala que el beneficio que representa para un autor contar con un perfil público en el repositorio de su institución es equivalente al beneficio que para una institución representa contar con un repositorio digital, en términos de ventaja competitiva, valor añadido, incremento de la visibilidad y facilidades de recuperación y acceso a la producción generada.

Argentina

Recibido: 31 de mayo de 2017 | Aceptado: 29 de agosto de 2017 | Publicado: 9 de octubre de 2017

Cita sugerida: Genovés, P. (2017). Perfiles de autor en repositorios institucionales. Palabra Clave (La Plata), 7(1), e033._https://doi.org/10.24215/18539912e033 


\section{Acceso abierto y repositorios institucionales}

El movimiento de acceso abierto (Open Access Movement, anteriormente conocido como Free Online Scholarship) es la iniciativa internacional surgida en la década de 1990 en el seno de la comunidad científica en respuesta a la crisis generada por la escalada de precios de las revistas científicas - con la consecuente imposibilidad de las instituciones de ciencia y técnica de seguir sosteniendo las suscripciones-, y al desafío de aplicar las últimas tecnologías de información y comunicación a la transformación y mejora del sistema de comunicación científica. Formalizado a inicios de este siglo mediante una serie de declaraciones públicas, tiene por objetivo promover la remoción de las barreras económicas y de algunas barreras legales que históricamente han restringido el acceso libre y gratuito a la literatura científica. Las dos estrategias inicialmente recomendadas a los científicos y académicos para lograrlo aún continúan vigentes, y son: el autoarchivo en archivos electrónicos abiertos (open archives), y la publicación en revistas de acceso libre y gratuito (open access journals) (Suber, 2015; Alonso Arévalo, Subirats Coll y Martínez Conde, 2008).

La estrategia del autoarchivo o ruta verde del acceso abierto (green $O A$ ) se debe a la Iniciativa de Archivos Abiertos (Open Archives Initiative) que, en 1999 y en vista del éxito de ArXiv, el repositorio de e-prints en física y ciencias relacionadas creado en 1991, desarrolló la tecnología necesaria para mantener archivos electrónicos interoperables mediante un protocolo de red, el OAI-PMH (Protocol Metadata Harvester). En el contexto OAI, al igual que en el contexto OA, el término archivo (archive) es sinónimo de repositorio de trabajos académicos (repository of scholary papers), pero el término abierto (open) refiere, ya no al acceso libre y gratuito a sus contenidos, sino a su cualidad de ser interoperable. Así, los repositorios son definidos en este contexto como aquellos servidores accesibles en red capaces de procesar correctamente peticiones OAI-PMH de proveedores de servicios (service providers), que usan los metadatos así recogidos para desarrollar servicios de valor agregado como, por ejemplo, una interfaz de búsqueda unificada (Alonso Arévalo, Subirats Coll y Martínez Conde, 2008; Carpenter, 2003).

Suele ser difícil distinguir entre repositorios, bibliotecas y archivos digitales, ya que muchas veces se usan los términos en forma indistinta, habida cuenta de las grandes semejanzas que existen entre los tres productos. En el contexto del acceso abierto, lo que caracteriza a un repositorio digital es justamente la aplicación del protocolo de interoperabilidad antes mencionado y del autoarchivo; luego, una característica compartida con las bibliotecas digitales son los servicios relativos al acceso público a través de un sitio web a sus colecciones digitales $y$, finalmente, en concordancia con la función principal de los archivos, su compromiso de preservación documental a largo plazo.

Mientras los distintos tipos de repositorios digitales comparten la misión de reunir, organizar, preservar y dar acceso a colecciones de objetos digitales, son las características de sus colecciones las que permiten distinguirlos. Así, los repositorios institucionales son aquellos que recogen la producción académico-científica generada por los miembros de una institución (académica, científica, gubernamental, etc.), los temáticos reúnen 
documentos generados por miembros de varias instituciones sobre una misma materia o disciplina, y los repositorios de datos recogen datos científicos generados durante las investigaciones (Alonso Arévalo, Subirats Coll y Martínez Conde, 2008; Barton y Waters, 2004).

Los repositorios institucionales tienen por objetivo "poner a disposición de la sociedad y del resto de investigadores toda la producción científica generada por la institución y hacer que ese contenido sea fácilmente recuperable y disponible en beneficio de la organización y del resto de la sociedad" (Alonso Arévalo, Subirats Coll y Martínez Conde, 2008, p. 30), y suponen para la propia institución "una ventaja competitiva (...) que proporciona un valor añadido, que es el acceso libre a su producción científica, a la vez que incrementa la visibilidad y el impacto propio y de sus investigadores" (Alonso Arévalo, Subirats Coll y Martínez Conde, 2008, p. 30). Mientras que para otros autores "tienen dos propósitos fundamentales: permitir (...) distribuir sus artículos de investigación gratis vía Internet, y asegurar la conservación de los mismos en un entorno electrónico y dinámico" (Barton y Waters, 2004, p. 8).

La tipología documental de los repositorios institucionales suele ser muy variada, encontrándose "desde productos netamente científicos elaborados en el ámbito de la propia institución o por su personal (tesis, libros y capítulos de libros, artículos de publicaciones periódicas, trabajos presentados a eventos, patentes), hasta documentación administrativa y normativa (reglamentaciones, memorias, documentos de archivo), incluyendo en algunos casos objetos de aprendizaje (guías y apuntes de clases), entre otros" (Pené, Unzurrunzaga y Borrell, 2015, p. 7). También puede encontrarse, por mandatos legales de depósito o bien acuerdos internos, la producción científica editada por la institución, esto es, números completos de revistas, libros y series monográficas, actas de reuniones, etc.

Los usos de los repositorios institucionales son también muchos y variados, desde la comunicación académica y la conservación de materiales digitales a largo plazo, hasta la organización de materiales de aprendizaje y de documentos de investigación, pasando por la gestión de metadatos y formatos y de herramientas de búsqueda y descubrimiento, entre otros (ARL, 2009; Alonso Arévalo, Subirats Coll y Martínez Conde, 2008; Barton y Waters, 2004).

A estos servicios básicos se agregan los llamados servicios de valor agregado o de valor añadido, creados en torno a la provisión de nuevos contenidos o bien de contenidos existentes provistos bajo nuevas formas, entre los que pueden mencionarse la provisión de datos estadísticos sobre consultas y descargas de documentos, la exportación de referencias a gestores de citas para el armado de CVs y bibliografías, los mapas conceptuales, los perfiles institucionales, la integración a redes sociales, etc. (ARL, 2009). Los servicios de valor añadido, en especial las estadísticas de uso, fueron tradicionalmente utilizados por los gestores de repositorios institucionales como estrategias de promoción del autoarchivo, atendiendo a la recomendación inicial del movimiento de acceso abierto. Sin embargo, mientras son una fortaleza de los repositorios temáticos, siguen siendo una carencia de los institucionales, probablemente porque los temáticos surgieron antes, sus colecciones tienen gran volumen y son más usados por los investigadores (Barrueco, 
2013). Cualquiera sea la razón, la importancia de los servicios de valor agregado para los repositorios digitales es clara: "Si queremos que los repositorios se conviertan en una pieza más del sistema de comunicación científica es necesario que sean percibidos como algo útil y valioso por parte de los investigadores" (Barrueco, 2013, p.14).

Lo que comenzó como una recomendación a los autores de depositar una copia de sus trabajos en archivos abiertos devino, dada la escasa respuesta recibida, en mandatos de depósito bajo distintas formas legales, desde resoluciones a nivel institución hasta leyes nacionales. Nuestro país formalizó tempranamente un mandato de depósito nacional: la Ley 26.899 Creación de Repositorios Digitales Institucionales de Acceso Abierto, Propios o Compartidos 1 , sancionada en 2013 y reglamentada tres años después, establece la obligatoriedad de los organismos e instituciones que componen el Sistema Nacional de Ciencia, Tecnología e Innovación (SNCTI) de desarrollar repositorios digitales institucionales en los que sus investigadores, docentes, estudiantes de maestría y doctorado deberán depositar una copia de la versión final de su producción científicotecnológica en un plazo no mayor a los seis meses desde la fecha de su publicación oficial o de su aprobación. En este contexto, la correcta identificación de los autores y de su producción almacenada en los repositorios digitales adquiere una importancia mayor.

\section{Identificadores y perfiles de autor}

En el ámbito de la actividad científica actual, donde la producción y el intercambio de conocimiento continúa incrementándose día a día e involucra en altísimo grado a los medios digitales, y donde además las publicaciones se encuentran almacenadas en una gran variedad de fuentes documentales, la correcta identificación de los autores, sus datos académicos y su producción se vuelve esencial, especialmente para el cálculo justo de indicadores cienciométricos, pero también para utilidad del propio investigador ya que hace a la visibilidad y reconocimiento de su trabajo en los medios digitales. Ambos elementos, identificadores y perfiles, están directamente relacionados con la identidad digital y la reputación científica de los autores y, por otro lado, con el control de autoridades gestionado tradicionalmente en las bibliotecas, igualmente necesario en los sistemas bibliográficos actuales, incluidos los repositorios digitales (Alonso Arévalo, 2014; Barrueco, 2013).

Si bien complementarios, identificadores y perfiles de autor son bien diferentes entre sí:

Por identificador de autor se entiende un código con forma numérica o alfanumérica que se asigna a un autor para identificar de forma inequívoca su producción científica, con independencia de cómo firma o en qué institución trabaja. El término perfil de autor se aplica al conjunto de datos que recogen de forma estandarizada bien únicamente su producción científica o toda su actividad investigadora (puestos desempeñados, proyectos de investigación, contratos, etc.) (Lorenzo Escobar y Pastor Ruiz, 2012, p.109).

Los perfiles de autor pueden o no estar ligados a un identificador de autor, y a su vez pueden $o$ no estar integrados a otros sistemas de comunicación científica. Para comprender los alcances y diferencias entre los sistemas dirigidos a la generación de 
identificadores y perfiles, se presenta un esquema de clasificación que distingue cuatro grupos de sistemas (Lorenzo Escobar y Pastor Ruiz, 2012):

- Sistemas de Identificación Puros: se limitan al desarrollo de un identificador; por ejemplo, ISNI (International Standard Name Identifier, estándar ISO 27729)르 e IraLis (International Registry of Authors-Links to Identify Scientists). 3

- Sistemas de Perfil Puros: se limitan al desarrollo de un formato de currículum normalizado sin asignarle ningún identificador; por ejemplo, los sistemas curriculares CVar de Argentina4 4 , LATTES $\underline{5}$ de Brasil, y CVN (Curriculum Vitae Normalizado) $\underline{6}$ de España.

- Sistemas Mixtos: permiten la asignación de un identificador y un perfil a cada autor; por ejemplo ResearchID y Scopus Author Identifier.

- Sistemas Globales: integran identificadores y perfiles generados por cualquier otro sistema; por ejemplo, ORCID (Open Researcher and Contributor Identifier) $\underline{Z}$ y VIVOㅁ.

Los sistemas de identificación puros se distinguen de los sistemas globales, o de los identificadores asociados a perfiles, en que su propósito no está ligado a la reunión de producción alguna, sino solamente a la identificación de personas, y serán otros sistemas los que, gracias a esta identificación, podrán establecer la relación de un investigador con su producción aplicando el identificador.

Los sistemas de perfil puros son equiparables a los Current Research Information Systems (CRIS) o Sistemas de Información Científica (SIC), aquellos sistemas de información que almacenan y gestionan datos acerca de las investigaciones que se realizan en una institución. Los tres ejemplos mencionados en este grupo, CVar, LATTES y CVN, son todos iniciativas nacionales; se trata de sistemas de información curricular en los que el perfil de cada investigador reúne en forma estructurada información personal y de contacto, producción científica y académica, cargos docentes y de investigación, etc.; en el caso de Brasil, además, su registro en el sistema es requisito para la evaluación y asignación de recursos. En el caso de nuestro país, el sistema CVar es parte del Sistema de Información de Ciencia y Tecnología Argentino (conocido por su sigla SICYTAR), una multiplataforma proveedora de datos sobre personas, grupos y proyectos de investigación y desarrollo, instituciones y equipamiento. Creado en 2011 por iniciativa de la Subsecretaría de Evaluación Institucional dependiente de la Secretaría de Articulación Científico Tecnológica del Ministerio de Ciencia, Tecnología e Innovación Productiva, el CVar provee actualmente perfiles de acceso público con información curricular, estadística y de contacto de todas aquellas personas que desempeñan actividades científicas y tecnológicas en instituciones argentinas, tanto públicas como privadas, y está en desarrollo la aplicación de un sistema de identificación única de autores (MINCYT, 2015). 
Los sistemas mixtos son iniciativas comerciales ligadas a productos bibliográficos; así, Scopus Author Identifier y ResearchID son sistemas que asignan identificadores y perfiles integrados a las bases de datos de literatura científica Scopus y Web of Science, respectivamente. La gran diferencia entre ellos es que ResearchID requiere que los autores se den de alta y gestionen su perfil, mientras que Scopus Author Identifier es aplicado por Scopus a todos los autores de documentos registrados en la base de datos; tampoco el sistema de perfiles Scopus Author Profile permite ser gestionado por los autores. Ambos sistemas tienen en común la generación de perfiles de autor conteniendo la producción almacenada en las bases de datos del sistema, junto a métricas de impacto basadas en citas y ligadas al contexto.

De los sistemas globales, ORCID es el sistema de identificación de autores orientado a la comunidad científica que se impone con más fuerza actualmente (García Gómez, 2012), dado que sus identificadores permiten enlazar todos los documentos de un autor $-y$ agregar información académica- independientemente de los sistemas bibliográficos en los que se encuentren registrados; es decir, su uso no queda ligado a ninguna plataforma o servicio bibliográfico particular. El objetivo de ORCID es integrar los distintos sistemas de identificación existentes (ResearchID, Scopus Author Identifier, etc.), a fin de que todos puedan seguir en uso, pero no en forma aislada. VIVO, por su parte, es una herramienta de descubrimiento basado en un sistema global de identificación de autores; su característica y diferencia con los restantes sistemas de identificación, incluido ORCID, es la descentralización de los datos de identificación. Sus datos son gestionados por universidades que, al participar del proyecto, los comparten con el conjunto de las universidades participantes a través de una aplicación desarrollada por VIVO y basada en la interoperabilidad semántica.

Google Citations $\underline{9}_{+}$el servicio de gestión de perfiles de Google Scholar, y las redes sociales científicas Academia.edu $\underline{10}$ y ResearchGate $\underline{11}$, son ejemplos de servicios de creación de perfiles que comparten con los sistemas de perfil puros la no inclusión de un sistema de identificación alfanumérica, y con los sistemas mixtos la integración a otras plataformas y servicios bibliográficos.

Google Scholar Citations, sin ser un sistema curricular en sentido estricto, permite al autor de textos académicos recogidos en el índice Google Scholar reunirlos en un perfil para hacer el seguimiento de las citas recibidas por artículo y contar, además, con métricas basadas en citas. Estos perfiles, además, pueden hacerse públicos, con lo cual son visibles para cualquier usuario del sistema y susceptibles de aparecer en los resultados de búsqueda.

Academia.edu y ResearchGate, por su parte, están igualmente orientados al ámbito educativo y científico pero, a diferencia de los sistemas de información curricular y del servicio de perfiles de Google Scholar, constituyen redes sociales científicas (Alonso Arévalo, 2014). Esto significa que la posibilidad de gestionar perfiles de investigación en estos sistemas está ligada a la formación de redes de colaboración entre investigadores, y es esa capacidad para compartir recursos entre colegas, enriquecida con la provisión de estadísticas de acceso, descarga y uso (cita) de la propia producción, y la integración con sistemas bibliográficos externos, lo que las convierte en un medio muy poderoso de 
visibilizar y potenciar los propios resultados de investigación. Esto explica finalmente el gran uso que de ellas se hace en los ámbitos científicos y educativos de todo el mundo.

\section{Perfiles de autor en repositorios institucionales}

La inclusión de perfiles de autor en repositorios institucionales surge inicialmente como un servicio de valor agregado destinado a promover la práctica del autoarchivo y el uso general del repositorio entre los miembros de una institución, dentro de una estrategia más amplia orientada al crecimiento y la sostenibilidad de los repositorios (Bongiovani, 2013). Al emular la utilidad de las redes sociales científicas, los perfiles institucionales (también llamados perfiles de investigador, hojas de vida, bibliografías de reconocimiento o perfiles de reconocimiento), constituyen un tipo de sistema de perfil puro que permite reunir la producción de un mismo autor disponible en el repositorio, enriqueciéndola con datos personales y de afiliación, estadísticas de productividad y uso y alertas, entre otras funcionalidades, además de proveer enlaces a otros identificadores y perfiles de sus autores.

En el contexto de los repositorios institucionales, mantener un perfil le permite al investigador gestionar mejor sus depósitos, acceder a todos sus documentos en forma estructurada, conocer los accesos y descargas que tuvieron, darlos a conocer fácilmente entre colegas, alumnos, etc. A su vez, en un ámbito académico, los perfiles permiten a los alumnos y docentes descubrir nuevos autores y documentos y acceder por otros caminos a los documentos generados en su propia institución (tesis escritas y dirigidas, programas de asignaturas y seminarios, libros y artículos publicados por sus colegas o profesores, etc.).

A pesar de sus ventajas, los perfiles de autor constituyen un servicio de valor añadido poco usado hasta ahora, según se desprende del análisis de contenido realizado en febrero 2017 sobre los sitios web de los primeros cincuenta (50) repositorios institucionales mejor puntuados en el Ranking Web de Repositorios $\underline{12}$. Los resultados indican que sólo 5 repositorios (10\%) proveen el servicio de perfiles de autor, los cuales se detallan a continuación:

\section{University of Pennsylvania ScholarlyCommons}

Software: Digital Commons

Perfil de ejemplo: https://works.bepress.com/julie fairman/

Contenidos del perfil:

- Documentos del autor registrados en el repositorio, agrupados por tipo documental.

- Breve presentación del autor señalando sus áreas de interés científico.

- Disciplinas en las que desarrolla sus actividades académicas y de investigación.

- Cargos actuales en la institución.

- CV descargable.

- Información de contacto. 
Digital Repository Iowa State University

Software: Digital Commons

Perfil de ejemplo: https://works.bepress.com/dean-adams/

Contenidos del perfil:

- Documentos del autor registrados en el repositorio, agrupados por tipo documental.

- Breve presentación del autor señalando sus áreas de interés científico.

- Disciplinas en las que desarrolla sus actividades académicas y de investigación.

- Cargos actuales en la institución.

- Títulos académicos.

- Enlaces a sitios web personales/institucionales.

- CV descargable.

- Información de contacto.

\section{Digital CSIC}

Software: Dspace

Perfil de ejemplo: https://digital.csic.es/cris/rp/rp03001

Contenidos del perfil:

- Documentos del autor registrados en el repositorio (accesibles como resultados de búsqueda). > Fotografía del autor.

- Firma en el repositorio. Otras firmas (nombres variantes).

- Centro o Instituto en que se desempeña. Departamento.

- Categoría profesional.

- Especialización (áreas de investigación).

- Enlaces a identificadores y perfiles del autor en otros sistemas (ORCID, Google Scholar, Scopus Author ID, ResearchGate, etc.)

- Enlaces a sitios web personales/institucionales.

- E-mail de contacto.

- Redes de colaboración (coautoría).

- Estadísticas de uso (vistas y descargas).

- Alertas por e-mail de nuevos documentos del autor.

- RSS.

\section{Utrecht University Repository}

Software: Dspace

Perfil de ejemplo: http://www.narcis.nl/person/RecordID/PRS1296312/id/10/Language/en Contenidos del perfil:

- Áreas de interés científico. Palabras claves. Disciplinas. Identificadores 
alfanuméricos del autor.

- Lugar de trabajo, enlace al sitio web y datos de contacto.

- Publicaciones más recientes registradas en el repositorio. Todas las publicaciones registradas.

- Proyectos de investigación finalizados.

- Clasificación temática.

- Redes de colaboración (coautoría).

Universidad de los Andes (Venezuela) Repositorio Institucional

Software: Dspace

Perfil de ejemplo: http://saber.ula.ve/handle/123456789/9686

Contenidos del perfil (generado como ítem bibliográfico)

- Resumen curricular.

- Categoría de investigador.

- Dependencia. Grupos de investigación.

- Grado académico. Título profesional.

- Líneas de investigación.

- E-mail. Teléfono.

- Fecha.

- Colecciones del repositorio en las que aparece registrada su producción científica.

- CV descargable.

- Documentos del autor registrados en el repositorio (accesibles como resultados de búsqueda).

Entre los repositorios institucionales argentinos, un análisis equivalente realizado en la misma fecha sobre los sitios web de los veintiocho (28) repositorios institucionales registrados en OpenDOAR $\underline{13}$ muestra que el servicio de perfiles de autor es aún menos frecuente, ya que lo proveen sólo dos (2) repositorios, y se detallan a continuación:

\section{CONICET Digital}

Software: DSpace

Perfil de ejemplo: http://ri.conicet.gov.ar/author/5770

Contenidos del perfil:

- Lugar de trabajo.

- Título y Grado.

- Categoría.

- Disciplina.

- Campo de aplicación.

- Especialidad. 
- Producción científico tecnológica del autor registrada en el repositorio.

\section{Memoria Académica}

Software: Greenstone

Perfil de ejemplo:http://www.memoria.fahce.unlp.edu.ar/perfiles/0729KarczmarczykP.html Contenidos del perfil:

- Fotografía del autor.

- Enlaces a identificadores y perfiles del autor en otros sistemas.

- Variantes del nombre.

- Grado académico.

- Departamentos. Unidades de investigación.

- Áreas de trabajo/estudio.

- Currículum abreviado.

- Producción disponible, agrupada por tipo documental.

- Estadísticas de uso (descargas mensuales).

Del análisis de los contenidos disponibles en los perfiles de ejemplo se desprende que son muchos los contenidos comunes a todos ellos, distinguiéndose Digital CSIC y Utrecht University Repository por los servicios añadidos, y Universidad de los Andes (Venezuela) Repositorio Institucional por aplicar un esquema Dublin Core Cualificado a la descripción de personas. En lo referido al software, 4 de los 7 repositorios recogidos utilizan DSpace, 2 Digital Commons y 1 Greenstone.

Este escenario de escasa aplicación del servicio de perfiles está cambiando paulatinamente hacia la integración de los repositorios con los sistemas de gestión de información científica institucionales. En este sentido, las primeras instituciones en integrar su repositorio con la información curricular de sus investigadores fueron la Universidad de Rochester 14 , la Universidad de Hong Kong 15 , y la Real Academia de Artes y Ciencias de los Países Bajos para su portal NARCIS 16 (COAR, 2013). A estos desarrollos puntuales se agrega DSpace-CRIS, un módulo adicional de DSpace -el principal software de código abierto para la gestión de repositorios-, dirigido a llevar a los repositorios funcionalidades propias de los CRIS. Esto significa que una institución puede gestionar, reunir y mostrar, desde el repositorio, todos los datos de investigación relativos a personas, unidades de investigación, proyectos, financiación, etc.; el módulo admite que los perfiles sean públicos o privados, a elección del autor, y que puedan ser creados tanto por los propios autores como por personal técnico del repositorio (CSIC, 2015 y 2017; Barrueco y Navalón, 2015).

La integración entre repositorios y CRIS, más allá de las tecnologías que lo hacen posible, es consecuencia natural de los profundos cambios que en el ámbito científico y académico vienen generando las tecnologías de información y comunicación en conjunción con la filosofía del acceso abierto, revolucionando lo que han sido sus prácticas tradicionales de comunicación, gestión y evaluación (Lorenzo Gil, Braña Ferreiro y Nieto Caramés, 2015; 
Martínez Galindo, Hernández San Miguel y Vallés Navarro, 2015); tiene además una relación directa con el establecimiento de mandatos institucionales y nacionales de depósito, el desarrollo de estándares y la conformación de redes nacionales, regionales e interregionales de repositorios.

\section{Consideraciones finales}

La actividad científica, generadora de conocimiento, tiene a la comunicación como motor principal, ya sea ésta informal (a través de contactos entre colegas, presentaciones a congresos, etc.), como formal (a través de la publicación de resultados de investigaciones). Mientras las tecnologías de información y comunicación están generando profundos cambios en todas las áreas de la actividad humana, en el ámbito científico están revolucionando lo que han sido sus prácticas tradicionales de comunicación formal, de la mano del movimiento de acceso abierto, pero también sus prácticas de comunicación informal, gracias al desarrollo de sistemas de comunicación e información de uso masivo, tanto dirigidos a científicos y académicos como al público en general. Los científicos y académicos están haciendo un uso cada vez mayor de plataformas y redes sociales para generar perfiles de autor, es decir, espacios virtuales donde difundir su currículum y contactar a otros colegas; espacios de apoyo a la construcción tanto de su reputación científica, mediante la visibilidad y las mediciones de impacto de su producción basadas en citas, como de su identidad digital, gracias a la asignación de identificadores asociados a sus datos personales y documentales en forma unívoca, y al uso de direcciones web permanentes.

En el contexto actual de la comunicación científica, caracterizada por el uso extendido de los medios digitales, los identificadores y perfiles de autor son herramientas complementarias que hacen a la identidad digital, otorgan visibilidad y sirven de apoyo a la reputación de los investigadores, al permitir conectar correctamente los documentos con sus autores, aun cuando presenten variaciones en las formas del nombre. Es justamente esa reunión bajo un mismo identificador lo que permite la generación de indicadores de producción e impacto que reflejen mejor su reputación, al incluir documentos que de otra manera quedarían fuera de las métricas.

La incorporación de las utilidades propias de las redes sociales científicas a los repositorios digitales dio lugar a los perfiles de autor como un servicio de valor agregado que reúne la producción de un autor almacenada en el repositorio, enriquecida con estadísticas de uso, información curricular y de contacto, etc. El beneficio que representa para un autor contar con un perfil público en el repositorio de su institución es equivalente al beneficio que para una institución representa contar con un repositorio digital, en términos de ventaja competitiva, valor añadido, incremento de la visibilidad y facilidades de recuperación y acceso a la producción generada.

Ante el establecimiento de mandatos nacionales de depósito, la necesidad de contar con repositorios institucionales fuertes y sostenibles en el tiempo es aún mayor que antes. Integrar a los repositorios las funcionalidades de los sistemas curriculares y de gestión de información científica y tecnológica es un camino para lograr esa fortaleza, y los perfiles de autor son el resultado visible de dicha integración. Por otra parte, al igual que con los 
repositorios en general, donde lo que comenzó como recomendaciones de autoarchivo devino en mandatos de depósito, con el servicio de perfiles de autor puede suceder que, planteado inicialmente como premio o incentivo al autoarchivo, se convierta en una herramienta de ayuda para controlar y certificar que se ha cumplido con la norma legal que obliga al depósito. Además, en aquellos repositorios que reúnen documentos editados por la institución (libros, revistas, actas de congresos, etc.) junto a los recibidos mediante autoarchivo, los perfiles de autor se convierten en la forma más adecuada y ventajosa de identificar la producción científica de los autores pertenecientes a la propia institución, y distinguirla del conjunto de documentos almacenados en el repositorio.

Por último, una consideración acerca de la expresión "valor agregado", aplicada a productos y servicios generados a partir del procesamiento de información ya existente, como es el caso de los perfiles institucionales: su uso deja al descubierto que en esencia se trata de un valor no añadido sino recobrado, un valor ya presente (latente) en dicha información, que sólo hace falta identificar y mostrar de manera significativa y ordenada.

\section{Notas}

1. Texto de la ley recuperado de:

http://repositorios.mincyt.gob.ar/pdfs/Boletin_Oficial_Ley_26899.pdf

2. http://www.isni.org/

3. http://www.iralis.org/es

4. Acceso a CVar desde el Portal de Información de Ciencia y Tecnología Argentino: http://datos.mincyt.gob.ar

5. http://lattes.cnpq.br/

6. https://cvn.fecyt.es/

7. https://orcid.org/

8. http://vivo.cornell.edu/

9. https://scholar.google.com/intl/es/scholar/citations.html

10. https://www.academia.edu/

11. https://www.researchgate.net/

12. http://repositories.webometrics.info/es/top_Inst_es

13. http://www.opendoar.org/index.html

14. https://urresearch.rochester.edu/home.action

15. http://hub.hku.hk/

16. https://www.narcis.nl 


\section{Referencias bibliográficas}

Alonso Arévalo, J. (Marzo 2014). Alfabetización en comunicación científica: acreditación, $\mathrm{OA}$, redes sociales, altmetrics, bibliotecarios incrustados y gestión de la identidad digital. En Encuentro Nacional de Bibliotecas Universitarias. Alfabetización informacional: Reflexiones y experiencias. Lima, Perú. Recuperado de http://eprints.rclis.org/22838/

Alonso Arévalo, J., Subirats Coll, I. y Martínez Conde, M. (2008). Informe APEI sobre acceso abierto. (Informe APEI; 2). Gijón: Asociación Profesional de Especialistas en Información. Recuperado de http://eprints.rclis.org/12507/1/informeapeiaccesoabierto.pdf

ARL Digital Repository Issues Task Force. (2009). The Research Library's Role in Digital Repository Services. Final Report. Washington: Association of Research Libraries. Recuperado de http://www.arl.org/storage/documents/publications/repository-servicesreport-jan09.pdf

Barrueco, J. (2013). RePEc o cómo proveer servicios de valor añadido a una comunidad de investigadores. Métodos de información (MEI), II Época, 4(6), 5-15. https://www.doi.org/10.5557/IIMEI4-N6-005015

Barrueco, J. y Navalón, J. (Marzo 2015). Desarrollo de perfiles de autores en DSpace para el repositorio institucional de la Universitat de València. En XIV Workshop Rebiun de Poyectos Digitales / VI Jornadas OS-Repositorios, Córdoba, España. Recuperado de http://helvia.uco.es/xmlui/handle/10396/12622

Barton, M. y Waters, M. (2004). Manual LEADIRS II. Cómo crear un repositorio institucional. Recuperado de https://www.recolecta.fecyt.es/sites/default/files/contenido/documentos/mit.pdf

Bongiovani, P. (Julio 2013). Crecimiento y sostenibilidad de los repositorios institucionales. En Repositorios digitales institucionales: diseño, implementación y optimización de un recurso estratégico para las universidades [curso modalidad virtual]. PICT-O CIN 2010149.

Carpenter, L. (2003). Open Archives Forum. Glossary. University of Bath. Recuperado de http://www.oaforum.org/tutorial/english/page6.htm

COAR. (2013). Incentivos, integración y mediación: prácticas sostenibles para poblar repositorios. Confederación de Repositorios de Acceso Abierto. Recuperado de https://www.coar-repositories.org/files/Sustainable-best-practices-spanish_final.pdf

CSIC. (2015). DIGITAL.CSIC Annual Report 2015. Recuperado de http://hdl.handle.net/10261/134937

CSIC. (2017). Guía para abrir perfiles de investigador en DIGITAL.CSIC. Oficina Técnica de DIGITAL.CSIC (versión 1) 15/02/2017. Recuperado de http://hdl.handle.net/10261/143953

García Gómez, C. (2012). Orcid: un sistema global para la identificación de investigadores. El profesional de la información, 21(2), 210-212.https://www.doi.org/10.5557/IIMEI4-N600501510.3145/epi.2012.mar. 14 
Lorenzo Escolar, N. y Pastor Ruiz, F. (2012). Un análisis de los principales sistemas de identificación y perfil para el personal investigador. Aula abierta, 40(2), 107-118. Recuperado de https://dialnet.unirioja.es/servlet/articulo?codigo $=3921021$

Lorenzo Gil, E., Braña Ferreiro, E. y Nieto Caramés, S. (Marzo 2015). Estudio de la integración de repositorios en el sistema científico-investigador: alternativas y estado actual. En XIV Workshop Rebiun de Proyectos Digitales / VI Jornadas OS-Repositorios, Córdoba, España. Recuperado de http://helvia.uco.es/xmlui/handle/10396/12631

Martínez Galindo, F., Hernández San Miguel, J. y Vallés Navarro, R. (Marzo 2015). RiuNet como nexo de integración de los contenidos de la UPV. En XIV Workshop Rebiun de Proyectos Digitales / VI Jornadas OS-Repositorios, Córdoba, España. Recuperado de http://helvia.uco.es/xmlui/handle/10396/12616

MINCYT (Octubre de 2015). Nuevo sitio del Sistema de Información de Ciencia y Tecnología Argentino. Recuperado de: http://www.mincyt.gob.ar/noticias/nuevo-sitio-delsistema-de-informacion-de-ciencia-y-tecnologia-argentino-11530

Pené, M. G., Unzurrunzaga, C. y Borrell, M. (2015). Repositorios institucionales universitarios argentinos, un acercamiento a sus colecciones. En IV Jornadas de Intercambio y Reflexión acerca de la Investigación en Bibliotecología, La Plata, Argentina. Recuperado de http://www.memoria.fahce.unlp.edu.ar/trab eventos/ev.5293/ev.5293.pdf

Suber, P. (2015). Acceso abierto. Toluca: Universidad Autónoma del Estado de México. Recuperado de http://ri.uaemex.mx/handle/20.500.11799/21710 